\title{
CORRIGENDUM
}

\section{The application of the first order system transfer function for fitting The California Verbal Learning Test Learning Curve-CORRIGENDUM}

Igor I. Stepanov, Charles I. Abramson, Oliver T. Wolf, AND Antonio Convit

doi:10.1017/S1355617709991457, Published online by Cambridge University Press, March 1, 2010.

The paper by Stepanov et al. (2010) has one typographical error that runs throughout the entire article that may be of some concern to readers.

It is stated throughout the paper that the version of the CVLT that was administered to the subject population was the CVLT-II. This is incorrect; the version administered was the CVLT.
This typographical error does not change the findings or the impact of the paper. Our modeling of the learning curve is equally valid for the original CVLT version [1] as well as for the CVLT-II [2].

\section{REFERENCE}

Stepanov, I.I., Abramson, C.I., Wolf, O.T., \& Convit, A. (2010). The application of the first order system transfer function for fitting the California Verbal Learning Test learning curve. Journal of the International Neuropsychological Society, 16(3), 443-452. 